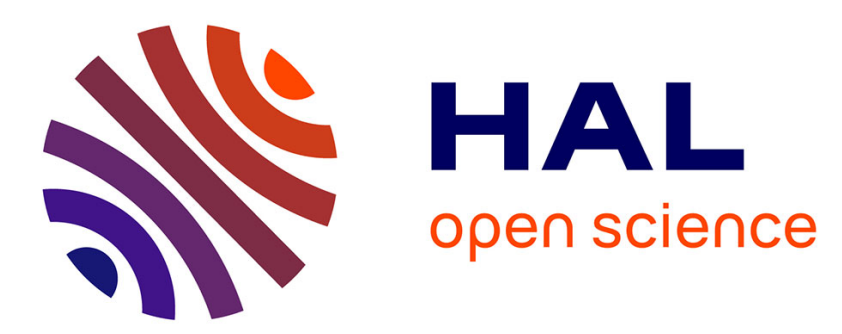

\title{
A game theoretic approach for the association problem in two-tier HetNets
}

\author{
Majed Haddad, Piotr Wiecek, Eitan Altman, Habib Sidi
}

\section{To cite this version:}

Majed Haddad, Piotr Wiecek, Eitan Altman, Habib Sidi. A game theoretic approach for the association problem in two-tier HetNets. ITC - 25th International Teletraffic Congress, Sep 2013, Shanghai, China. pp.1-9, 10.1109/ITC.2013.6662962 . hal-00913200

\section{HAL Id: hal-00913200 \\ https://hal.inria.fr/hal-00913200}

Submitted on 10 Dec 2013

HAL is a multi-disciplinary open access archive for the deposit and dissemination of scientific research documents, whether they are published or not. The documents may come from teaching and research institutions in France or abroad, or from public or private research centers.
L'archive ouverte pluridisciplinaire HAL, est destinée au dépôt et à la diffusion de documents scientifiques de niveau recherche, publiés ou non, émanant des établissements d'enseignement et de recherche français ou étrangers, des laboratoires publics ou privés. 


\title{
A Game Theoretic Approach for the Association Problem in Two-Tier HetNets
}

\author{
Majed Haddad*, Piotr Wiecek ${ }^{\dagger}$, Eitan Altman* and Habib Sidi ${ }^{\ddagger}$ \\ ${ }^{*}$ INRIA Sophia-Antipolis, 10 route des Lucioles, 06902 Sophia-Antipolis, France \\ ${ }^{\dagger}$ Institute of Mathematics and Computer Science, Wroclaw University of Technology, Poland \\ ${ }^{\dagger}$ CERI/LIA, University of Avignon, Agroparc BP 1228, Avignon, France
}

\begin{abstract}
This paper addresses a Bayesian game theoretic framework for determining the association rules that decide to which cell a given mobile user should associate in LTE two-tier Heterogeneous Networks (HetNets). Users are assumed to compete to maximize their throughput by picking the best locally serving cell with respect to their own measurement, their demand and a partial statistical channel state information (CSI) of other users. In particular, we investigate the properties of a hierarchical game, in which the macro-cell BS is a player on its own. We derive analytically the utilities related to the channel quality perceived by users to obtain the equilibria. We show by means of a Stackelberg formulation, how the operator, by dynamically choosing the offset about the state of the channel, can optimize its global utility while end-users maximize their individual utilities. The proposed hierarchical decision approach for wireless networks can reach a good trade-off between the global network performance at the equilibrium and the requested amount of signaling. Typically, it is shown that when the network goal is orthogonal to user's goal, this can lead the users to a misleading association problem. Numerical results validate the expectation from the theoretical analysis and illustrate the advantages of the proposed approach.
\end{abstract}

Index Terms-4G LTE macro-cell, small-cells, dynamic offset, association problem, channel state information, game theory, Bayes-Nash equilibrium, Bayes-Stackelberg equilibrium, price of anarchy.

\section{INTRODUCTION}

In 4th generation (4G) systems with full frequency reuse, there is an increasing demand for higher user and system throughput, along with growing expectation for all mobile stations (MSs) in a cell to be available to multimedia and internet services. This is especially difficult to maintain at the celledge, where received signal and service clearly deteriorate. Consequently, future wireless networks are evolving towards heterogeneous architectures, where in each cell a user may have available multiple different types of access points (APs) (e.g., small-, pico-, femto-cells, relays) [1]. Intuitively, this has many positive effects for a MS resulting from the improved spatial reuse of resources. However, small-cell overlays also imbue many difficulties, e.g., cell-organisation/optimisation, resource allocation and interference coordination. While smallcells represent an efficient solution to provide local capacity enhancements, they can not replace macro cells which ensure area coverage and support highly mobile terminals. Hence, a two-tier architecture for cellular systems naturally emerges which poses the challenge of how small-cells and macro-cells can coexist.

From the system design perspective, the given model is very useful in practice. In LTE networks, UEs associate typically with the cell with highest Received Signal Reference Power (RSRP). RSRP is a measure of the received signal strength of a cell at a user equipment (UE) and it is measured based on the strength of certain reference signals that cells broadcast [2]. Recently, papers like [3] raises the cell reselection process problem in HetNets. Clearly, the association based on highest signal strength is inadequate to address this challenge. Moreover, in the 3GPP RRC standard [4] in Section 5.1.5 it is clearly said that the E-UTRAN can configure a list of cell specific offsets and a list of blacklisted cells. Typically, Release 8 UEs should apply the ranking based on radio link quality (with offsets) unless operator indicates support for prioritybased reselection. This suggests that the offset can be configured for each and every cell depending on the priority set by the operator (e.g., mobility, requested throughput, signalling).

Altogether, these issues motivate the need for an alternative approach for determining the association rules that decide to which cell UEs should associate. Later in the paper, we will raise the question of dynamic offset design and configuration to address these challenges.

Efficient design of wireless networks calls for end users implementing radio resource management (RRM), which requires knowledge of the mutual channel state information in order to limit the influence of interference impairments on the decision making. However, full CSI assumption is not always practical because communicating channel gains between different users in a time varying channel within the channel coherence time may lead to large overhead. In this case, it is more appropriate to consider each channel coherence time as a one-stage game where players are only aware of their own channel gains and their opponent's channel statistics (which vary slowly compared to the channel gains and, therefore, can be communicated [5]). The interaction between the players may be repeated but with a different and independent channel realization each time and therefore is not a repeated game. This motivates the use of games with incomplete information, also known as Bayesian games [6], [7] which have been incorporated into wireless communications for problems such as power control [8], spectrum management in the interference channel [9] and load balancing in multi- 
technology system [10]. In [8], a distributed uplink power control in a multiple access (MAC) fading channel was studied and shown to have a unique Nash equilibrium (NE) point. With the same incomplete information, it was shown [9] that in a symmetric interference channel with a one-time interaction, there exists a unique symmetric strategy profile which is a NE point. This result however is limited to scenarios where all users statistically experience identical channel conditions (due to the symmetry assumption) and does not apply to interactions between weak and strong users.

In this paper, we propose a Stackelberg formulation of the association problem when a partial channel state information is assumed at the transmitter. By Stackelberg we mean distributed decision making assisted by the network, where the wireless users aim at maximizing their own utility, guided by aggregated information broadcasted by the network (or the macro-cell) about the CSI of each user. This information is referred to as channel quality indicator thresholds in the rest of the paper and represents the dynamic offset set by the operator. We first show how to derive the utilities of users that are related to their respective channel quality under the different association policies. We then derive the policy that corresponds to the Stackelberg equilibrium and compare it to the centralized and the non-cooperative model. Technically, our approach not only aims at improving the network equilibrium efficiency but has also two nice features: (i) It allows the network to guide users to a desired equilibrium that optimizes its own utility if it chooses the adequate information to send, (ii) Only the individual user demand and a partial statistical CSI of other users is needed at each transmitter. Our approach contributes to designing networks where intelligence is split between the macro-cell base station (BS) and mobile stations in order to find a desired trade-off between the global network performance reached at the equilibrium and the amount of signaling needed to make it work.

The structure of the paper is as follows. The system model related aspects are described in Sec. II. Section III defines users' utilities. Next, we provide a thorough analysis of the Bayes equilibria for both non-cooperative and Stackelberg frameworks: Sec. IV presents the non-cooperative game and Sec. V presents the Stackelberg Bayesian game framework adopted for the considered association problem. We first show how the macro-cell BS can control the equilibrium of its users by means of a Stackelberg formulation and then we derive analytically the utilities of the users and compute equilibria. We also present three different evaluation scenarios along with some key performance indicators including price of anarchy. In Sec. VI, we provide numerical results to illustrate the theoretical solutions derived in the previous sections. Sec. VII concludes the paper.

\section{SySTEM MOdEL}

Consider a heterogeneous wireless system consisting of a single MAN (Metropolitan Area Network) 4G LTE macro-cell and a set of partly overlapping LAN (Local Area Networks) small-cells. Each user arriving in the system will decide individually to which of the available systems it is best to connect according to its radio condition, its demand and the statistical information about other users. Their strategies are then based on this (incomplete) information. The association problem is then generalized to allow the macro-cell BS to control the users' behavior by broadcasting appropriate information, expected to maximize its utility while individual users maximize their own utility.

$b_{i}$ is the demand of user $i\left(b_{i}=1\right.$ when there exists a demand, and $b_{i}=0$ otherwise) and $a_{i}$ his action defined by the user decision to connect to a certain radio access technology (RAT). $a_{i}=1$ when the user chooses the smallcell, and $a_{i}=0$ when the user chooses 4G LTE. $h_{i}$ is the downlink channel power gain between the small-cell access point and the mobile terminal. We statistically model the signal amplitude $\sqrt{h_{i}}$ as a random variable with a Rayleigh distribution ${ }^{1}$. By the transformation theorem for single random variable, it is well known that the effect of Rayleigh fading on power attenuation is equivalent to consider the channel power gain $h_{i}$ as an exponentially distributed random variable with mean $\lambda_{i}$ [11]. We will see later how $\lambda_{i}$ is related to different parameters adopted throughout the paper. We assume that the user state is defined by the pair $\left(h_{i}, b_{i}\right)$. The network is fully characterized by the user state. However, when distributing the joint radio resource management (JRRM) decisions, this complete information is not available to the users. The macrocell BS and the small-cell AP broadcasts to its terminals an aggregated information indicating a measurement of the communication quality of the wireless channel (excellent, fair, poor...). This can be done through the Channel Quality Indicator (CQI) which can be a value (or values) representing a measure of channel quality for a given channel. Typically, a high value CQI is indicative of a channel with high quality and vice versa. More formally, assume that the knowledge of each user about his own state is limited to the pair $\left(s_{i}, b_{i}\right)$, where $s_{i}=\mathbb{I}_{\left\{h_{i}>\Psi_{i}\right\}}$, with $\Psi-$ a fixed threshold and $\mathbb{I}_{C}$ is the indicator function equal to 1 if condition $C$ is satisfied and to 0 otherwise. We will call $\Psi_{i}$ the "CQI threshold" of user $i$ which represents the dynamic offset parameter set by the maco-cell. Thus, a user only knows whether he wants to transmit and whether the channel is in a good $\left(s_{i}=1\right)$ or in a bad $\left(s_{i}=0\right)$ condition given the CQI threshold. In addition any player has the information about the probability distribution of his own state $\left(s_{i}, b_{i}\right)$ and that of his opponent $\left(s_{j}, b_{j}\right)$. These are given by $\alpha_{i}$ - the probability to have $\left\{h_{i}>\Psi_{i}\right\}$, and $\beta_{i}$ - the probability that $b_{i}=1$.

In the next sections, we provide a thorough analysis of the existence and characterization of the Bayes equilibria for both non-cooperative and Stackelberg scenarios.

The first step before analyzing the Stackelberg Bayesian decision scheme is to define the utilities of users. These are often related to throughput, whose variations are mainly due to network load, radio network conditions and mobility such as handovers.

\footnotetext{
${ }^{1}$ The method can be extended to other fading distributions too.
} 


\section{A. 4G LTE Throughput}

We consider a Real-time Transport Protocol (RTP) streaming service. As we consider cellular networks where Adaptive Modulation and Coding (AMC) ensure that the Block Error Rate (BLER) is lower than a certain target, the video quality is guaranteed when the throughput required by the codec is obtained. The goal of a streaming user is thus to achieve the best throughput, knowing that the different codecs allow a throughput between an upper (best) $v_{\max }$ and a lower (minimal) $v_{\min }$ bounds (where $v_{\max }>v_{\min }$ ). His utility is expressed by the quality of the streaming flow he receives, which is in turn closely related to his throughput. Indeed, a streaming call with a higher throughput will use a better codec offering a better video quality. This throughput depends not only on the peak throughput, but also on the evolution of the number of calls in the system where the user decides to connect. Note that a user that cannot be offered this minimal throughput in neither of the available systems is blocked in order to preserve the overall network performance. However, once connected, we suppose that a call will not be dropped even if its radio conditions degrade because of mobility.

Assuming proportional fair scheduling among different users, the throughput of a user connected to the 4G LTE macro-cell when there are $k$ users connected is given by:

$$
v=\min \left[D \frac{G}{\sum_{i=1}^{k}\left(1-a_{i}\right) b_{i}}, v_{\max }\right]
$$

where $G$ is the opportunistic scheduler gain and $D$ is the $4 \mathrm{G}$ LTE peak rate (i.e., the throughput he would obtain if he were alone in the cell). Note here that the admission control will ensure that $v \geq v_{\min }$ by blocking new arrivals. We may assume also that $D G \geq v_{\max }$.

\section{B. The small-cell Throughput}

The measurement of average throughput of a node in a small-cell is done by the time it takes to transfer the files between the AP and the wireless clients. The throughput depends on the bit rate at which the wireless mobile communicates to its AP, which greatly depends on the attenuation level at the receiver side due to his geographical position. This can vary greatly depending on the link conditions. The small-cell throughput can thus be expressed by

$$
T h p_{i}^{W}=\log \left(1+\frac{p h_{i} a_{i} b_{i}}{\sigma^{2}}\right)
$$

where index $W$ stands for the small-cell network, $\sigma^{2}$ is the noise variance and $p$ the user's transmit power. We further assume that there is no interference between 4G LTE macrocell and the small-cell.

Given $\lambda_{i}$ and $\Psi_{i}$ we can compute that the distribution of $h_{i}$ is $\operatorname{Exp}\left(\lambda_{i}\right)$ with

$$
\alpha_{i}=\exp \left(-\lambda_{i} \Psi_{i}\right)
$$

Knowing the information that a player has, there are four possible policies of a player $i$ with $b_{i}=1$ (we do not consider state $b_{i}=0$, when there is no transmission of any type):

\begin{tabular}{l||l|l|l|l}
$h_{i}<\Psi_{i}$ & $W$ & $W$ & $C$ & $C$ \\
\hline$h_{i}>\Psi_{i}$ & $W$ & $C$ & $W$ & $C$
\end{tabular}

where index $C$ stands for the macro-cell network. Let us not consider the policy $(W, C)$, which is irrational, as the throughput of a player using the small-cell when $\left\{h_{i}>\Psi_{i}\right\}$ is certainly higher than that when $\left\{h_{i}<\Psi_{i}\right\}$. We then have a game with partial CSI with two states and 3 actions for each player in every state.

We assume that the information about the channel quality that user $i$ possesses is limited to that about the distributions of states $\left(s_{j}, b_{i}\right)$ of each of the players (including $\left.i\right)$, that is about $\alpha_{j}$ (or $\lambda_{j}$ ) and $\beta_{j}$ and to exact information about his own current state $\left(s_{i}, b_{i}\right)$ (but not about exact value of $h_{i}$ ). We make two additional assumptions about the model considered. The first one is that the model is symmetric, that is all the values $\beta_{i}, \lambda_{i}$ and $\Psi_{i}$ defining it, are the same for each of the players (and equal to $\beta, \lambda$ and $\Psi$ respectively). The second one is that $v_{\max } \geq 2 v_{\min }$. Both of them aim at simplifying the notation used in our considerations. On the other hand we believe that some counterparts of all our results are true also without these assumptions, so they can be made without serious limitation of generality.

\section{UTILITIES}

We assume that each of the players uses one of the three policies $W W, C W, C C$, where first letter stands for a player's action when his channel is bad, and the second one when his channel is good. As it is troublesome to write down the policies for each of $n$ players, we will make use of the fact that the game is symmetric, writing instead of the policy profile a policy statistics $\mathbf{K}=\left[k_{C C}, k_{C W}\right]$ with $k_{C C}$ denoting the number of players applying policy $C C$ and $k_{C W}$ - of players applying $C W$. Of course the number of those using policy $W W$ is $n-k_{C C}-k_{C W}$, so we will omit it. Given $\mathbf{K}$, we can define user $i$ 's utility in state $s=0,1 \mathrm{as}^{2}$

$$
u_{i}(s, \mathbf{K})= \begin{cases}v_{\mathbf{K}_{-i}} ; & \text { if user } i \text { chooses } C \text { at state } s, \\ C(s) ; & \text { if user } i \text { chooses } W \text { at state } s\end{cases}
$$

where the functions $C(s)$ describing the utility using the smallcell, are defined as follows;

$$
\begin{gathered}
C(1)=[c(h) \mid h>\Psi]=\frac{1}{\alpha} \int_{\Psi}^{\infty} c(h) \lambda e^{-\lambda h} d h, \\
C(0)=[c(h) \mid h<\Psi]=\frac{1}{1-\alpha} \int_{0}^{\Psi} c(h) \lambda e^{-\lambda h} d h,
\end{gathered}
$$

$c(h)$ above is the utility using $W$ when channel gain is $h$ and is defined as follows:

$$
c(h)=\log \left(1+\frac{p h}{\sigma^{2}}\right)
$$

${ }^{2}$ Notation $\mathbf{K}_{-\mathbf{i}}$ used above denotes policy statistics without policy of user 
The values $v_{\mathbf{K}_{-i}}^{i}$, describing the utility of player $i$ using 4G LTE when his opponents use policies described by $\mathbf{K}$ are given in Eqt. (8) where $k^{*}=\max \left\{k: \frac{D G}{k} \geq v_{\min }\right\}$.

The utility expression reflects the fact that some arrivals may be blocked by the admission control when there is not enough resource for all the players - in that case we assume that some $k$ players (where $k$ is such that $\frac{D G}{k} \geq v_{\text {min }}$ ) willing to connect to 4G LTE network are chosen at random (with equal probabilities) and they receive the service ${ }^{3}$.

\section{THE NON-COOPERATIVE EQUILIBRIUM}

Game theory has accentuated the importance of randomized games or mixed games. However, such a game does not find any significant role in most communication modems or source coding codecs since equilibria where each user randomly picks a decision at each time epoch cannot be used effectively there, as they amount to perpetual handover between networks.

Definition 1 (Bayes-Nash equilibrium). A strategy profile $\mathbf{P}_{\mathbf{i}}{ }^{B N E}, \forall i=1,2$ corresponds to a Bayes-Nash equilibrium (BNE) if, for all users, any unilateral switching to a different strategy cannot improve user's payoff at any state. Mathematically, this can be expressed by the following inequality, given the statistical information about the other user $\forall \mathbf{Q}_{\mathbf{i}} \neq \mathbf{P}_{\mathbf{i}}{ }^{B N E}$

$u_{i}\left(s_{i},\left(\mathbf{P}_{\mathbf{i}}^{B N E}, \boldsymbol{P}_{-i}^{B N E}\right)\right) \geq u_{i}\left(s_{i},\left(\mathbf{Q}_{\mathbf{i}}, \boldsymbol{P}_{-i}^{B N E}\right)\right) ;$ for $s_{i}=0,1$

Proposition 1. The symmetric $n$-user game considered in the paper always has a pure-strategy Bayes-Nash equilibrium of one of seven types:

(a) When $v_{[k-1, l]} \geq C(1) \geq v_{[k, l-1]} \geq C(0) \geq v_{[k, l]}$, then any profile where $k$ players use policy $C C, l$ players use policy $C W$, and all the others play $W W$ is an equilibrium.

(b) When $v_{[k-1,0]} \geq C(1) \geq C(0) \geq v_{[k, 0]}$ then any profile where $k$ players apply policy $C C$ and the remaining $n-k$ players use policy $W W$ is an equilibrium.

(c) When $v_{[k-1, n-k]} \geq C(1) \geq v_{[k, n-k-1]} \geq C(0)$ then any profile where $k$ players apply policy $C C$ and the remaining $n-k$ players use policy $C W$ is an equilibrium.

(d) When $v_{[n-1,0]} \geq C(1)$ then the profile where all the players use policy $C C$ is an equilibrium.

(e) When $C(1) \geq v_{[0, k-1]} \geq C(0) \geq v_{[0, k]}$, then any profile where $k$ players use policy $C W$ and all the others play $W W$ is an equilibrium.

(f) When $C(1) \geq v_{[0, n-1]} \geq C(0)$ then the profile where all the players use policy $C W$ is an equilibrium.

(g) When $C(0) \geq v_{[0,0]}$ then the profile where all the players use policy $W W$ is an equilibrium.

We give a corollary to this proposition. It gives a kind of consistency property for equilibria in games for different values of $n$.

\footnotetext{
${ }^{3}$ In reality the ones chosen at random in our model would be those whose request was considered first.
}

\section{Corollary 1.}

(a) Suppose that a profile where at least one player uses policy $W W$ and the number of players using policies $C C$ and $C W$ is $k$, is an equilibrium in n-user symmetric game. Then it is also an equilibrium in any $m$-user game defined with the same parameters $\beta, \lambda$ and $\Psi$ and $m \geq k$.

(b) Moreover for any fixed parameters $\beta, \lambda$ and $\Psi$ there exists an $n$ such that for any $m>n$ at least $m-n$ players use policy $W W$ in any equilibrium in $m$-user game.

Proof: Note that $v_{[k, l]}$ does not depend on the number of players in the game $n$, only on the number of those who use one of the policies $C W$ or $C C$. Just this implies part (a). Part (b) is due to the fact that $v_{[k, l]} \rightarrow 0$ as either $k \rightarrow \infty$ or $l \rightarrow \infty$.

\section{THE HIERARCHICAL EQUILIBRIUM}

In this section, we propose a methodology that transforms the above non-cooperative game into a Stackelberg game. Concretely, the macro-cell may guide users to an equilibrium that optimizes its own utility $U_{B S}$ if it chooses the adequate information to send. The macro-cell BS utility, defined as the expected throughput of 4G LTE network, can be written as in (10).

We exemplify our general analysis by investigating the possibility of considering three scenarios for the choice of $\Psi$ :

1) Centralized model - the macro-cell BS chooses both $\Psi \mathrm{s}$ and the policies for the players, aiming to maximize $U_{B S}$.

2) Stackelberg model - there are two stages: at the first one the macro-cell BS chooses both $\Psi$ given the information about the distributions of $(h, b)$ aiming to maximize the throughput of the macro-cell at the second stage, when players play the game from the last section. The proposed approach can be seen as intermediate scheme between the centralized model and the fully non-cooperative model,

3) Fully non-cooperative model - the game has two stages: at the first one, players choose their $\Psi$ given the information they have about the distributions of $(h, b)$ aiming to maximize their expected throughput at the second stage; at the second stage they choose a policy depending on actual $(s, b)$ as in the model of the last section.

We consider all the above scenarios, yet in scenario 3) we only show that the equilibrium is symmetric, but assume that players may act asymmetrically in general. To do so we look for symmetric equilibria in the model (which exist, but we believe are not the only ones possible there, even though the model itself is symmetric). The rationale behind this simplification is twofold: firstly - considering asymmetric equilibria would cause various problems with notation; secondly and most important - we believe that asymmetric equilibria, where users may have different functional form of their strategies, are harder to justify, as they would require prior coordination among the devices to agree on which equilibrium is played. 
$v_{[k, l]}=\sum_{r=0}^{k} \sum_{q=0}^{l} \sum_{w=0}^{q} \beta^{r+q}(1-\beta)^{k+l-r-q}\left(\begin{array}{c}k \\ r\end{array}\right)\left(\begin{array}{c}l \\ q\end{array}\right)\left(\begin{array}{c}q \\ w\end{array}\right) e^{-\lambda(q-w) \Psi}\left(1-e^{-\lambda \Psi}\right)^{w} \frac{\min \left\{k^{*}, r+w+1\right\}}{r+w+1} \min \left\{\frac{D G}{\min \left\{k^{*}, r+w+1\right\}}, v_{\max }\right\}$

$U_{B S}([k, l], \Psi)=\sum_{r=0}^{k} \sum_{q=0}^{l} \sum_{w=0}^{q} \beta^{r+q}(1-\beta)^{k+l-r-q}\left(\begin{array}{c}k \\ r\end{array}\right)\left(\begin{array}{l}l \\ q\end{array}\right)\left(\begin{array}{c}q \\ w\end{array}\right) e^{-\lambda(q-w) \Psi}\left(1-e^{-\lambda \Psi}\right)^{w} \min \left\{D G, v_{\max }(w+r)\right\}$

\section{Proposition 2.}

1) In the centralized model, the macro-cell BS chooses any value of $\Psi$ and $C C$ policies for all the users.

2) In the Stackelberg model, the macro-cell BS computes

$$
C(\infty)=\int_{0}^{\infty} c(h) \lambda e^{-\lambda h} d h
$$

and finds $k^{* *}$ such that

$$
v_{\left[k^{* *}-1,0\right]}>C(\infty) \geq v_{\left[k^{* *}, 0\right]}
$$

$\operatorname{Next}^{4}$ :

(a) If $n \leq k^{* *}$ then at the equilibrium the macro-cell BS chooses any $\Psi$ such that $v_{[n-1,0]} \geq C(1)(\Psi)$, and all the players use policy $C C$.

(b) If $n>k^{* *}$ then:

b1) for any $k$ such that $k^{* *} \leq k \leq n$ and any $0 \leq$ $l \leq k$ the macro-cell BS does the following steps: If $k=n$ it sets $\bar{\Psi}=0$, otherwise it finds $\bar{\Psi}$ such that

$$
C(0)(\bar{\Psi})=v_{[l, k-l]}(\bar{\Psi})
$$

If such a $\bar{\Psi}$ does not exist, it puts $P(k, l)=0$. Otherwise it finds $\underline{\Psi}$ such that

$$
C(1)(\underline{\Psi})=v_{[l, k-l-1]}(\underline{\Psi}) .
$$

If such a $\underline{\Psi}$ does not exist, it puts $\underline{\Psi}=0$. Finally it takes $\Psi(k, l)=\max \{\bar{\Psi}, \underline{\Psi}\}$ and computes

$$
P(k, l)=U_{B S}([l, k-l], \Psi(k, l))
$$

b2) it chooses $k_{\max }$ and $l_{\max }$ with the biggest value of $P(k, l)$ (which equals the BS utility at equilibrium). The choice of $\Psi\left(k_{\max }, l_{\max }\right)$ at the first stage and any profile of policies where $l_{\max }$ players use policy $C C$ and $k_{\max }-l_{\max }$ play $C W$ will then be an equilibrium.

3) In the fully non-cooperative model, the players choose $\Psi^{*}$ satisfying

$$
c\left(\Psi^{*}\right)=v_{[0, n-1]}\left(\Psi^{*}\right)
$$

\footnotetext{
${ }^{4}$ In what is written above, we use the convention that $C(s)(\Psi)$ means the value of $C(s)$ when CQI threshold is $\Psi$. Similarly $v_{\mathbf{K}}(\Psi)$ is the value of $v_{\mathbf{K}}$ when his opponent's threshold is $\Psi$.
}

and then all use $C W$ policies at the second stage of the game.

For the clarity of the exposition, proofs are given in the Appendix. We give one corollary to this proposition by evaluating the price of anarachy (PoA). The PoA measures how good the system performance is when users play selfishly and reach the BNE instead of playing to achieve the social optimum.

Corollary 2. The price of anarchy in the n-user hierarchial model can be computed as

$$
P_{o} A=\frac{U_{B S}([n, 0], \Psi)}{U_{B S}(\mathbf{K}, \Psi)}
$$

(where $U_{B S}([n, 0], \Psi)$ is the maximum value of the macro-cell $B S$ 's utility obtained in scenario 1) of Proposition 2), which is independent of $\Psi$. Moreover:

1) In the Stackelberg model it is either equal to 1 when $k \leq k^{* *}$, or satisfies

$$
\operatorname{PoA}=\min _{k^{* *} \leq k \leq n, 0 \leq l \leq k} \frac{U_{B S}([n, 0], \Psi)}{P(k, l)}
$$

with $k^{* *}$ and $P(k, l)$ defined as in Proposition 2.

2) In the fully non-cooperative model it can be computed as:

$$
P_{o} A=\frac{U_{B S}([n, 0], \Psi)}{U_{B S}\left([0, n], \Psi^{*}\right)}
$$

with $\Psi^{*}$ defined as in Proposition 2.

The corollary is again just a rewriting of the results from Proposition 2 with the stress made on network utilities rather than strategies of the players. It shows that exactly the same procedure, used to find the equilibrium policies, can be applied to evaluate the performance of the network.

\section{NUMERICAL RESUlTS}

We consider a scenario of an operator providing subscribers with a service available through a large 4G LTE cell coexisting with a small-cell access point. We first consider a streaming service where users require a minimal throughput of $250 \mathrm{kbps}$ and can profit from throughputs up to $2 \mathrm{Mbps}$ in order to enhance video quality $\left(v_{\min }=0.25 \mathrm{Mbps}\right.$ and $v_{\max }=2$ Mbps). As mentioned before, users are characterized by the distribution of their small-cell downlink channel and the distribution of their demand. We consider for each user an 


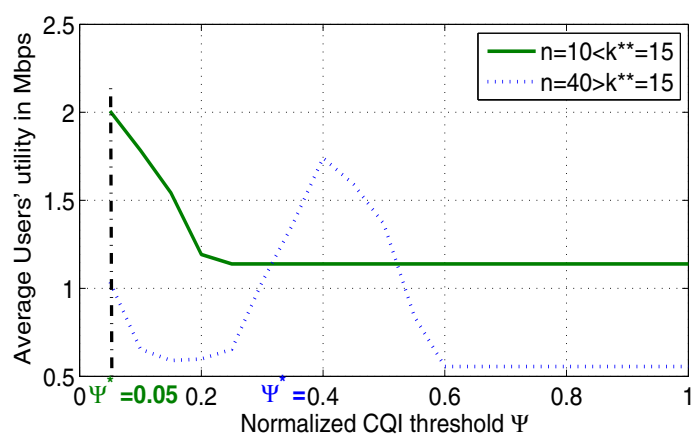

Fig. 1. Average users' utility for $n=40>k^{* *}=15$.

exponentially distributed channel fading with parameter $\lambda$ as defined in (3), assumed to be symmetric for the multi-users case. In order to validate our theoretical findings, we obtain users' actions at the equilibrium defined by users decisions to connect to a small-cell or 4G LTE at low and high channel state. In particular, we present extensive results for the hierarchical (Stackelberg) equilibrium, non- cooperative (Nash) equilibrium and compare them with the centralized strategy. To do so, we define a set of $n \in\{2, \ldots, 40\}$ competing users with Rayleigh fading characterized by parameter $\lambda$. For the following set of simulations we take $\lambda=4 d B$ with a line of sight channel gain of $20 \mathrm{~dB}$. Each user thus experience an average channel gain at $20 \%$ of the maximum transmission channel gain. We also set the demand load $\beta=0.5$ for every user and the channel state $\alpha$ derives from $\lambda$ and $\Psi$ as in Eqt. (3). 50 scenarios are simulated to remove the random effects from Rayleigh fading. To show the influence of user's CQI threshold $\Psi$ on the different equilibrium strategies, we compute the users' best responses for different values of the threshold $\Psi$. It is then possible to compute the non-cooperative BayesNash equilibrium strategies and the related users' utilities obtained at the equilibrium. For the hierarchical Stackelberg equilibrium, given the action of the BS macro-cell, i.e., the CQI threshold $\Psi$, we compute the best-response function of the mobile users, i.e., the action of the mobile users which maximizes their utilities given the action of the BS macrocell. The network utility is defined as the average throughput obtained by a user selecting the $4 \mathrm{G}$ LTE macro-cell. Finally, under the formerly defined policy statistics $\mathbf{K}=\{k, l\}$, the macro- and small-cell load $\mathcal{L}$ can be respectively expressed as follow:

$$
\begin{gathered}
\mathcal{L}(C)=(k+l \cdot \alpha) / n \\
\mathcal{L}(W)=(n-k-l \cdot \alpha) / n
\end{gathered}
$$

\section{A. Utilities}

Figure 1 depicts the average users' utility as function of the CQI threshold $\Psi$. This figure exhibits an optimum value of the CQI threshold $\Psi^{*}=0.4$. This is due to the fact that either very low or very high values of $\Psi$ give little information for the users about actual channel condition, which is what they want to avoid.

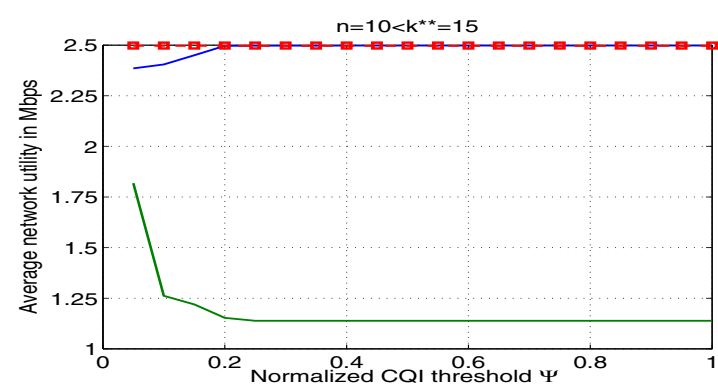

(a)

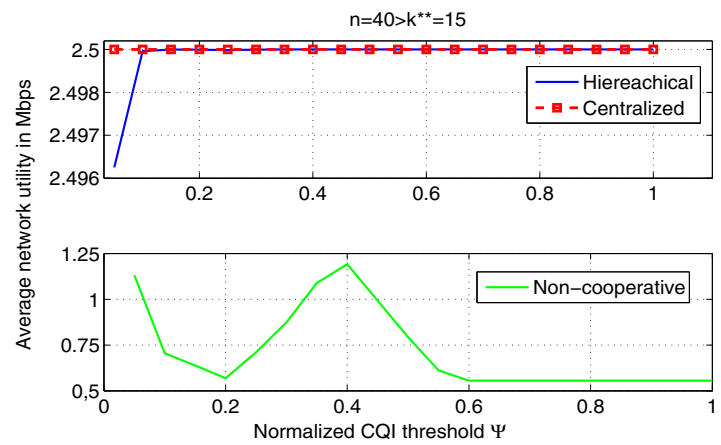

(b)

Fig. 2. Average network utility in function of CQI threshold $\Psi$. Cases $n \leq k^{* *}$ and $n \geq k^{* *}$.

In Fig. 2, we plot the network utility according to different values of $\Psi$ for the three, non-cooperative, hierarchical and centralized, approaches. In Fig. 2(a) we consider a number of interacting users $n \leq k^{* *}$ and in Fig. 2(b) $n \geq k^{* *}$. It is clearly seen that the hierarchical model exhibits merely the same performance than the centralized model thanks to an adequate choice of $\Psi$ contrary to the non-cooperative model which presents a bad network utility especially when $n>k^{* *}$. The reason behind this is that the information given to the user is misleading since the utility of the user cannot be directly inferred from the quality of his channel when the macro-cell goal is orthogonal to users' goal.

What we see in these figures is that when the macro-cell BS can decide on the behavior of the users (Stackelberg case), it forces them to use 4G LTE. In other cases (when users can decide on their behavior, but are given only partial information), the users' interest is to choose the CQI threshold somewhere in the middle of the channel gain range $\left(\Psi^{*}=0.4\right)$.

\section{B. Strategies}

In Figure 3(a), we plot the load of each RAT according to users strategies at the equilibrium as function of the normalised CQI threshold $\Psi$. First, as claimed by Prop. 21 ), we find that, for the centralized policy, all users choose policy $C C$ for any value of $\Psi$ fixed by the macro-cell BS. Second, in accordance to the result in Prop. 2 the load of the macro-cell and the small-cell load match the announced equilibrium policies for some specific values of the CQI threshold.

We find that increasing the value of $\Psi$, users have more incentive to choose strategy $C$ at the equilibrium. Asymptot- 


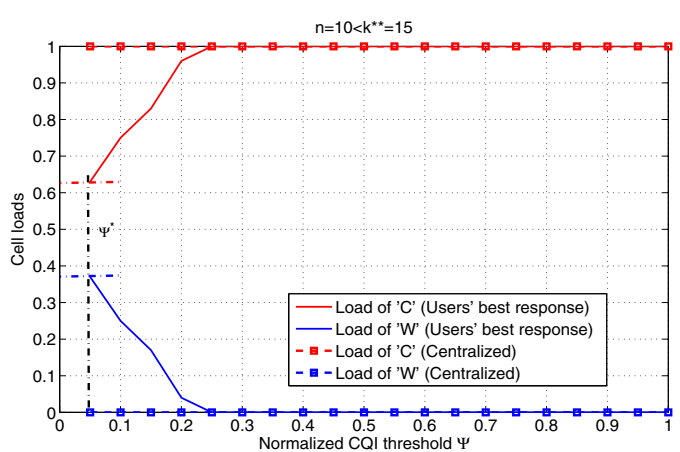

(a)

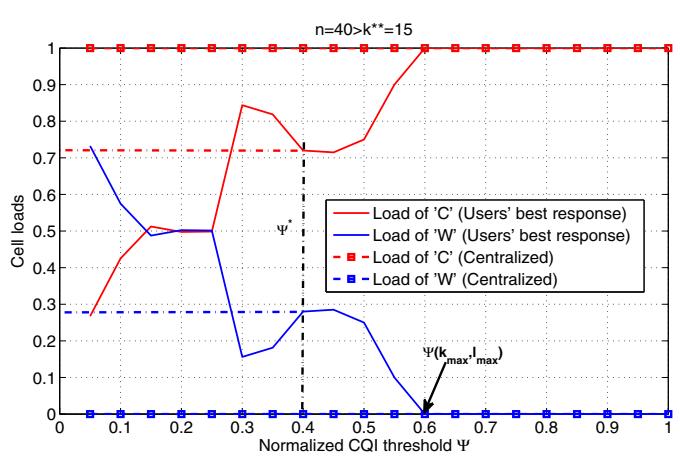

(b)

Fig. 3. Cell loads at equilibrium compared to centralized policy as function of $\Psi$.

ically, when $\Psi$ grows large, users choose policy $C C$ at the equilibrium.

Particularly, we observe for the case $n<k^{* *}$ in Figure 3(a) that the non-cooperative equilibrium is reached for $\Psi^{*}=$ 0.05 . This value of $\Psi^{*}=0.05$ is obtained by simulation (see Figure 1) and maximizes the average utility of users in the system which defines the non-cooperative equilibrium whereas the hierarchical equilibrium is reached for any value of $\Psi>$ 0.25 as Prop. 2 points out.

Conversely, in Figure 3(b) (case $n>k^{* *}$ ) we observe that for high values of $\Psi$ users rather select policy $C C$, and this indeed validates our result for the hierarchical equilibrium. Result from Figure 3(b) also matches with the strategies profiles at the non-cooperative equilibrium where users first select the CQI threshold $\Psi^{*}=0.4$ (from Proposition 2 2)(b)) which here also maximizes the average users utility (see Figure 1) and then select between strategies $C C$ and $C W$. Note that compared to the results pointed out by Proposition 23 ) there is a deviation of about $30 \%$ of expected $C W$ policy players toward $C C$ policy.

On the other hand, in the hierarchical model the macro-cell BS has an incentive to choose high values $\left(\Psi\left(k_{\max }, l_{\max }\right)=\right.$ 0.6) of CQI thresholds (see Figure 3(b)). The obtained value of $\Psi\left(k_{\max }, l_{\max }\right)=0.6$ cross the definition of the stackelberg equilibrium in Sec. V (the macro-cell average utility is already maximized at $\Psi=0.6$ from Figure 2(b)) with the announced properties in Prop. 2 by simulation. Numerically and according to the proposition, $\Psi\left(k_{\max }, l_{\max }\right)=0.6$ is obtained at $k_{\max }=$ 40 and $l_{\text {max }}=33$ here the average load of each cell is $\mathcal{L}(C)=$

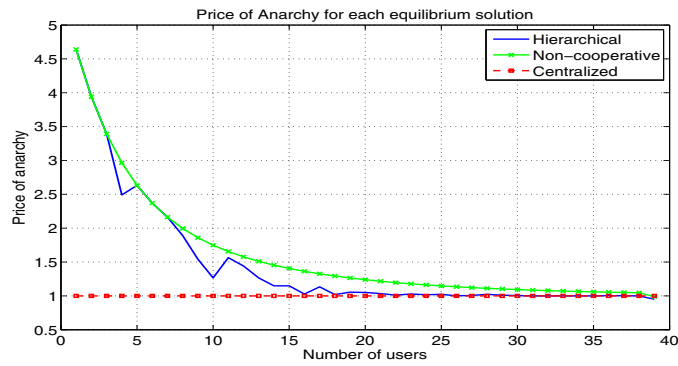

Fig. 4. The price of anarchy for increasing number of competing users $n$.

0.92 and $\mathcal{L}(W)=0.08$.

\section{Price of Anarchy}

In Figure 4, we observe as expected that the price of anarchy is non-increasing function of the number of users. This is a consequence of the fact that adding each new player to the game gives the macro-cell BS more patterns of behavior of the users which can be stimulated by a proper choice of $\Psi$. This result may seem surprising at first glance, as usually a bigger number of players means more anarchy. However, if we look at the objective function of the macro-cell BS in (10), which is the probability of all the players using 4G LTE network, we clearly see that a bigger number of players is disadvantageous for the small-cell network which may get congested and favorable for 4G LTE which cannot. Moreover, Figure 4 illustrates the fact that the PoA of the non-cooperative case is decreasing to 1 faster than the hierarchical case. The latter result offers hope that such a robust and accurate modeling can be designed around competition, because Stackelberg behavior does not arbitrarily degrade the mechanism's performance like the selfish does.

\section{CONCLUSION}

We have proposed a Bayesian Stackelberg association method for two-tier heterogeneous networks that combines benefits from both decentralized and centralized design. The macro-cell network operator optimizes its global utility while users maximize their individual utilities. The users' decision making is based on partial information that is signaled to the mobiles by the macro-cell BS. In this setting, we have shown that, in order to maximize its revenue, the network operator rather than increasing its offered throughput (which is costly) has an incentive to dynamically configure the offset (which is referred to as channel quality indicator thresholds in the analysis) neither very low nor very high. This may make the information given to the user when attempting to connect misleading since the throughput of a user cannot be directly inferred from the quality of his channel but also depends on the channel quality indicator thresholds the macro-cell BS fixes. We finally have characterized a global performance indicator of the network, namely the price of anarchy. It is shown that the proposed approach provides a reasonable trade-off between centralized vs decentralized optimization in terms of the signaling overhead and the resulting network throughput performance. 


\section{REFERENCES}

[1] J. G. Andrews, H. Claussen, M. Dohler, S. Rangan, and M. C. Reed, "Femtocells: Past, Present, and Future," Selected Areas in Communications, IEEE Journal on, vol. 30, no. 3, pp. 497-508, Apr. 2012.

[2] S. Sesia, I. Toufik, and M. Baker, LTE - The UMTS Long Term Evolution: From Theory to Practice, 2nd ed. Wiley, August 2011.

[3] S. Deb, P. Monogioudis, J. Miernik, and J. P. Seymour, "Algorithms for enhanced inter cell interference coordination (eicic) in lte hetnets," Accepted to appear in IEEE/ACM Transactions on Networking, 2013.

[4] "3rd generation partnership project; technical specification group radio access network; evolved universal terrestrial radio access (e-utra) radio resource control (rrc); protocol specification (release 8)," vol. 3GPP TS 36.331 V8.0.0, 2007.

[5] A. Leshem and E. Zehavi, "Cooperative game theory and the gaussian interference channel," IEEE Journal on Selected Areas in Communications, pp. 1078-1088, 2008.

[6] J. C. Harsanyi, "Games with incomplete information played by Bayesian players, I-III,' Manage. Sci., vol. 14, no. 3, pp. 160-182, 1967.

[7] D. Fudenberg and J. Tirole, Game Theory. MIT Press, 1991.

[8] G. He, M. Debbah, and E. Altman, "A bayesian game-theoretic approach for distributed resource allocation in fading multiple access channels," EURASIP J. Wirel. Commun. Netw., vol. 2010, pp. 8:1-8:12, January 2010.

[9] S. Adlakha, R. Johari, and A. Goldsmith, "Competition in wireless systems via bayesian interference games," arXiv:0709.0516v1, 2007.

[10] M. Haddad, S. E. Elayoubi, E. Altman, and Z. Altman, "A hybrid approach for radio resource management in heterogeneous cognitive networks," IEEE Journal on Selected Areas in Communications Special Issue on Advances in Cognitive Radio Networking and Communications, vol. 2, no. 6, pp. 733-741, April 2010.

[11] J. Li, A. Bose, and Y. Q. Zhao, "Rayleigh flat fading channels' capacity," in Proceedings of the 3rd Annual Communication Networks and Services Research Conference, May 2005.

\section{A. Proof of Proposition 1}

Before we prove Proposition 1, we need an auxiliary lemma.

Lemma 1. The functions $v_{[k, l]}(\Psi)$

(a) are decreasing in $k, l$,

(b) satisfy $v_{[k+1, l]}(\Psi) \leq v_{[k, l+1]}(\Psi)$,

(c) are nonincreasing in $\Psi$.

Proof: First define

$$
\begin{aligned}
F(r, q):= & \sum_{w=0}^{q}\left(\begin{array}{c}
q \\
w
\end{array}\right) e^{\lambda(q-w) \Psi}\left(1-e^{\lambda \Psi}\right)^{w} \frac{\min \left\{k^{*}, r+w+1\right\}}{r+w+1} . \\
& \min \left\{\frac{D G}{\min \left\{k^{*}, r+w+1\right\}}, v_{\max }\right\} .
\end{aligned}
$$

We start by showing that $F$ is decreasing both in $r$ and $q$ and that $F(r, a-r)$ is decreasing in $r$ for any fixed $a$. Define

$$
F_{0}(r, w)=\frac{\frac{\min \left\{k^{*}, r+w+1\right\}}{r+w+1} D G}{\min \left\{\frac{\min \left\{k^{*}, r+w+1\right\}}{\operatorname{mon}}, v_{\max }\right\}}
$$

For $w \leq k^{*}-r, F_{0}(r, w)=\min \left\{\frac{D G}{r+w+1}, v_{\max }\right\}$, which is clearly decreasing both in $w$ and $r$. Similarly, for $w \geq k^{*}-r$, $F_{0}(r, w)=\frac{k^{*}}{r+w+1} \min \left\{\frac{D G}{k^{*}}, v_{\max }\right\}$, which is also decreasing both in $w$ and $r$. Now note that $F(r, q)$ is the expected value of $F_{0}$ when $w$ is a random value with the binomial distribution $\operatorname{Bin}\left(q, 1-e^{-\lambda \Psi}\right)$. As $\operatorname{Bin}\left(q+1,1-e^{-\lambda \Psi}\right)$ strictly stochastically dominates $\operatorname{Bin}\left(q, 1-e^{-\lambda \Psi}\right)$, the expected value with respect to $\operatorname{Bin}\left(q+1,1-e^{-\lambda \Psi}\right)$ of any decreasing function is smaller than that with respect to $\operatorname{Bin}\left(q, 1-e^{-\lambda \Psi}\right)$ and thus

$$
F(r, q+1)<F(r, q) .
$$

To see that $F$ is also decreasing in $r$, it is enough to see that $F(r+1, q)$ and $F(r, q)$ are both expected values of
$F_{0}(r, w)$ when $w$ is a random value distributed according to the same distribution $\operatorname{Bin}\left(q, 1-e^{-\lambda \Psi}\right)$, and so they preserve the monotonicity of $F_{0}$.

Finally, to see that $F(r, a-r)$ is decreasing in $r$, note that it is the expected value of $F_{0}(r, w-r)=$ $\frac{\min \left\{k^{*}, w\right\}}{w} \min \left\{\frac{D G}{\min \left\{k^{*}, w\right\}}, v_{\max }\right\}$ when $w-r$ is distributed according to $\operatorname{Bin}\left(a-r, 1-e^{-\lambda \Psi}\right)$. The rest of the proof follows analogously to that of (19).

Now, to prove (a) of the lemma note that $v_{[k, l]}$ is the expected value of $\sum_{q=0}^{l} \beta^{q}(1-\beta)^{l-q}\left(\begin{array}{l}l \\ q\end{array}\right) F(r, q)$ when $r$ is a random value with the binomial distribution $\operatorname{Bin}(k, \beta)$. If we apply the same reasoning as the one leading to (19), we can show that $v_{[k, l]}$ is decreasing in $k$. The fact that it is also decreasing in $l$ is proved analogously - the only difference is that we need to write $v_{[k, l]}$ as $\sum_{r=0}^{k} \beta^{q}(1-\beta)^{k-r}\left(\begin{array}{c}k \\ r\end{array}\right) F(r, q)$, and then use the monotonicity of $F$ in $q$ (instead of the monotonicity in $r$ ).

To prove (b) first note that for any $k, l \geq 0$

$$
v_{[k, l]}=\sum_{p=0}^{k+l} \beta^{p}(1-\beta)^{k+l-p}\left(\begin{array}{c}
k+l \\
p
\end{array}\right) G(p, k, l),
$$

where $G(p, k, l)=\sum_{a=\max \{0, p-l\}}^{\min \{p, k\}} \frac{\left(\begin{array}{c}k \\ a\end{array}\right)\left(\begin{array}{c}l \\ p-a\end{array}\right)}{\left(\begin{array}{c}k+l \\ p\end{array}\right)} F(a, p-a)$. Note however that $G(p, k, l)$ is the expected value of $F(a, p-a)$ when $a$ is a random variable with the hypergeometric distribution Hypergeometric $(k+l, k, p)$. Since Hypergeometric $(k+l+1, k+1, p)$ strictly stochastically dominates Hypergeometric $(k+l+1, k, p)$, and $F(a, p-a)$ is a decreasing function of $a$ for any fixed $p, G(p, k+1, l)$, which is the expected value of $F(a, p-a)$ with respect to that first distribution is not bigger than $G(p, k, l+1)$, which is the expected value of $F(a, p-a)$ with respect to the second one. But this immediately implies that also

$$
\begin{aligned}
v_{[k+1, l]}= & p \sim \operatorname{Bin}(k+l+1, \beta)[G(p, k+1, l)] \\
\leq & p \sim \operatorname{Bin}(k+l+1, \beta)[G(p, k, l+1)] \\
& =v_{[k, l+1]}
\end{aligned}
$$

To prove part (c) of the lemma take $\Psi_{1}<\Psi_{2}$ and note that $F(r, q)(\Psi)$ is the expected value of $F_{0}$ when $w$ is a random value with the binomial distribution $\operatorname{Bin}\left(q, 1-e^{-\lambda \Psi}\right)$. Since $\operatorname{Bin}\left(q, 1-e^{-\lambda \Psi_{2}}\right)$ stochastically dominates $\operatorname{Bin}\left(q, 1-e^{-\lambda \Psi_{1}}\right)$, $F(r, q)\left(\Psi_{1}\right) \geq F(r, q)\left(\Psi_{2}\right)$. The rest of the proof follows along the same lines as the proof of part (a).

Now we are able to prove Proposition 1.

Proof:

It is enough to check the definition of Bayes-Nash equilibrium inferring $C(1)>C(0)$, and Lemma 1 if needed that the sets of inequalities appearing in the proposition define respective equilibria. What is left to show is that cases (a-g) cover all the possible situations. Suppose none of the cases e)-g) holds. Then either there exists a $l$ such that

$$
v_{[0, \hat{l}-1]} \geq C(1) \geq C(0) \geq v_{[0, \hat{l}]}
$$

which by (b) of Lemma 1 implies that

$$
v_{[0, \hat{l}-1]} \geq C(1) \geq C(0) \geq v_{[1, \hat{l}-1]}
$$


or

$$
C(1)<v_{[0, n-1]}
$$

In this case, again by (b) of Lemma 1, one of two possibilities is true:

$$
C(1) \leq v_{[n-1,0]}
$$

or there exists $\hat{k}$ such that

$$
v_{[\hat{k}, n-\hat{k}-1]} \leq C(1) \leq v_{[\hat{k}-1, n-\hat{k}]}
$$

The first possibility is covered by case (d) of the proposition. In the second one there are two subcases: when $v_{[\hat{k}, n-\hat{k}-1]} \geq$ $C(0)$, the condition of case (c) is satisfied. When $v_{[\hat{k}, n-\hat{k}-1]}<$ $C(0)$ we obtain the following inequalities:

$$
v_{[\hat{k}, n-\hat{k}-1]}<C(0) \leq C(1) \leq v_{[\hat{k}-1, n-\hat{k}]}
$$

which by (a) of Lemma 1 implies that

$$
v_{[\hat{k}, n-\hat{k}-1]}<C(0) \leq C(1) \leq v_{[\hat{k}-1, n-\hat{k}-1]} .
$$

Now suppose (22) or (27) holds (and put $\hat{l}=n-\hat{k}$ in the second case). Then by (b) of Lemma 1 for every $l<\hat{l}$ there exist $\underline{k}(l), \bar{k}(l) \leq n-\hat{l}$ such that

$$
v_{[\underline{k}(l), l]} \leq C(1) \leq v_{[\underline{k}(l)-1, l]}, \quad v_{[\bar{k}(l), l]} \leq C(0) \leq v_{[\bar{k}(l)-1, l]}
$$

If for every $l, \underline{k}(l)=\bar{k}(l)$, then also

$$
v_{[\underline{k}(0), 0]} \leq C(0) \leq C(1) \leq v_{[\underline{k}(0)-1,0]}
$$

and thus the inequalities of case (b) of the proposition are satisfied. Otherwise there exists the smallest $l$ such that

$$
v_{[\underline{k}(l), l]} \leq C(0) \leq C(1) \leq v_{[\underline{k}(l)-1, l]} .
$$

But for such an $l$, since by (a) and (b) of Lemma 1 , there has to be $v_{[\bar{k}(l)-1, l-1]} \geq C(1)$ and $v_{[\underline{k}(l)+1, l-1]} \leq C(0)$, consequently $\underline{k}(l)=\underline{k}(l-1)=\bar{k}(l-1)-1$ and thus $C(0) \leq$ $v_{[\underline{k}(l), l-1]} \leq C(1)$, which together with (29) implies that the conditions of case (a) of the proposition (with $k=\underline{k}(l)$ ) are satisfied, ending the proof.

\section{B. Proof of Proposition 2}

Proof:

Part 1) is obvious.

2) Since when $\Psi \rightarrow \infty, C(1)(\Psi) \rightarrow C(\infty)$, then if $v_{[n-1,0]}>C(\infty)$, for $\Psi$ large enough also $v_{[n-1,0]} \geq$ $C(1)(\Psi)$, which means that all the players apply policy $C C$ in equilibrium at the second stage of the game. Thus whenever $\Psi$ is big enough, the outcome of the Stackelberg game is that all the players use $4 \mathrm{G}$ LTE with probability 1 , which gives the biggest value possible of the base station's utility.

Now suppose that $v_{[n-1,0]} \leq C(\infty)$. Then for any value of $\Psi$, not every player uses policy $C C$ at the equilibrium of the game of the second stage. Thus, to maximize the $U_{B S}$, the macro-cell BS has to choose the $\Psi$ in such a way that at the equilibrium of the game of the second stage some (say $l$ ) players would apply policy $C C$ and some other (say $k-l$ ) would apply $W C$, and that the macro-cell BS's utility was the highest possible. This is done by solving the optimization problems of finding the smallest $\Psi$ such that the profile $[k-$ $l, l]$ is an equilibrium in the game defined by this $\Psi$, that is satisfying

$$
\begin{aligned}
v_{[l-1, k-l-1]}(\Psi) & \geq C(1)(\Psi) \geq v_{[l, k-l-1]}(\Psi) \\
& \geq C(0)(\Psi) \geq v_{[l, k-l]}(\Psi)
\end{aligned}
$$

if $k<n$ or

$$
v_{[l-1, n-l]}(\Psi) \geq C(1)(\Psi) \geq v_{[l, n-l-1]}(\Psi) \geq C(0)(\Psi)
$$

otherwise. However, as by Lemma $1, v_{\mathbf{K}_{-\mathbf{i}}}(\Psi)$ are decreasing functions of $\Psi$ for any fixed $\mathbf{K}$, while $C(s)(\Psi)$ are clearly increasing in $\Psi$, this maximum is achieved for $\Psi$ satisfying (13)-(14) (with modifications that when (13) does not have any solutions, there are no solutions to the above set of inequalities, while when (14) does not have a solution, the above inequalities are always true). When the values of $U_{B S}$ for each such $\Psi$ are computed, and the biggest one of them is chosen, this is certainly the biggest value of the macro-cell BS's utility that can be obtained in the Stackelberg scenario.

3) First note that whenever $\Psi^{*}$ is chosen as in (16), all the players choose $C W$ in equilibrium. This is because $C(0)$ is a conditional expectation of $c^{i}\left(h_{i}\right)$ over the set $H_{-}:=\left\{c^{i}\left(h_{i}\right) \leq\right.$ $\left.v_{[0, n-1]}\right\}$, so it is definitely smaller than $v_{[0, n-1]}$. Similarly, $C(1)$ is a conditional expectation of $c^{i}\left(h_{i}\right)$ over the set $H_{+}:=$ $\left\{c^{i}\left(h_{i}\right) \geq v_{[0, n-1]}\right\}$, so it is bigger than $v_{[0, n-1]}$. Thus the condition for the profile of all the players using $C W$ to be an equilibrium is definitely satisfied.

Now note that whenever player $i$ chooses $\Psi_{i}<\Psi^{*}$ at the first stage, but continues to use policy $C W$ in the second, he loses

$$
\int_{\Psi_{i}}^{\Psi^{*}}\left(v_{[0, n-1]}-c^{i}\left(h_{i}\right)\right) \lambda_{i} e^{-\lambda_{i} h_{i}}>0 .
$$

Similarly, when he chooses $\Psi_{i}>\Psi^{*}$, he loses

$$
\int_{\Psi^{*}}^{\Psi_{i}}\left(c^{i}\left(h_{i}\right)-v_{[0, n-1]}\right) \lambda_{i} e^{-\lambda_{i} h_{i}}>0 .
$$

On the other hand, when he changes both the $\Psi_{i}$ and the policy at the second stage, his utility is either $v_{[0, n-1]}$ (when he plays $C C$ ) or $\left[c^{i}\left(h_{i}\right)\right]$ (when he uses policy $W W$ ), which are clearly both less than his current utility

$$
P\left(h_{i} \in H_{-}\right) v_{[0, n-1]}+P\left(h_{i} \in H_{+}\right) \quad\left[c^{i}\left(h_{i}\right) \mid h_{i} \in H_{+}\right],
$$

so $\Psi^{*}$ is an equilibrium choice for each of the players.

The last thing we need to show is that there is $\Psi^{*}$ satisfying (16). See however that the function

$$
f(\Psi):=c^{i}(\Psi)-v_{[0, n-1]}(\Psi)
$$

is clearly (by (c) of Lemma 1) increasing, with $f(0)=-v_{\max }$ and $f \rightarrow \infty$ as $\Psi \rightarrow \infty$. It is also continuous (as both $c^{i}$ and $v_{[0, n-1]}$ are obviously continuous), so by the intermediate value property there exists a $\Psi^{*}$ satisfying $f\left(\Psi^{*}\right)=0$, which is equivalent to (16). 